\title{
Le rôle des jonctions intercellulaires dans le fonctionnement de la barrière hémato-testiculaire
}

Le testicule est constitué de deux compartiments anatomiquement et fonctionnellement distincts : les tubules séminifères et le tissu interstitiel. Ce dernier est le tissu remplissant les espaces entre les tubules et contenant, entre autres, les cellules de Leydig qui sont responsables de la production de testostérone. Les tubules sont de petits tubes creux, d'environ $250 \mu \mathrm{m}$ de diamètre. L'intérieur de chaque tube est tapissé d'un épithélium appelé "séminifère " dont la fonction essentielle est de produire les spermatozoïdes à l'issue de la spermatogenèse. Ce qui différencie les cellules germinales les plus mûres, les spermatozoïdes, des cellules somatiques, c'est le nombre de leurs chromosomes qui a été réduit de moitié au cours de la méiose. La méiose, ou division cellulaire réductionnelle, s'effectue durant la seconde moitié de la genèse des spermatozoïdes ou spermatogenèse.

\section{L'épithélium séminifère}

La méiose et l'achèvement de la spermatogenèse s'effectuent dans l'épithélium séminifère. Celui-ci est constitué de deux types cellulaires distincts : les cellules germinales et les cellules de Sertoli. Celles-ci nourrissent et soutiennent les cellules germinales au cours de leur développement. Les cellules de Sertoli cessent de se diviser chez l'adulte. Elles ont environ $70 \mu \mathrm{m}$ de long et sont comparables à un «arbre» dont le "tronc " serait fixé à la membrane limitante du tubule séminifère et dont les multiples "branches" entoureraient chaque cellule germinale, pour reprendre une image proposée par Elftman [1]. Des jonctions cellulaires sont présentes sur ce tronc de la cellule de Sertoli ; ces jonctions permettent à chaque cellule de Sertoli d'être reliée au tronc de cellules de Sertoli voisines. Trois types de jonctions y sont présentes qui sont définies par l'épaisseur de l'espace intercellulaire : la jonction serrée ou étroite (tight junction) mais que nous appellerons ici jonction occlusive, c'est-à-dire "qui ferme" l'espace intercellulaire, la jonction lacunaire (gap junction ou nexus) et la jonction adhérente (adhering junction). Par convention, cet espace est de plus de $10 \mathrm{~nm}$ dans les jonctions adhérentes, d'environ 2-4 nm dans les jonctions lacunaires et inexistant dans les jonctions occlusives où les feuillets externes des membranes plasmiques de cellules adjacentes semblent fusionnés. Ces jonctions coexistent le long de la membrane plasmique et forment ensemble le complexe jonctionnel de la cellule de Sertoli. L'intérêt de ce complexe de jonction réside en ce que les jonctions occlusives, qui en font partie, constituent une véritable barrière anatomique à l'entrée de substances provenant du sang dans le tubule séminifère [2-4].

\section{Les jonctions occlusives}

Tout au long de l'épithélium séminifère, le tronc de chaque cellule de Sertoli est réuni au tronc de la cellule de Sertoli voisine par une jonction occlusive qui forme une bande imperméable et continue autour de chaque cellule. Dès lors, une barrière hématologique est ainsi créée le long de chaque tubule séminifère du testicule : c'est la barrière hémato-testiculaire. Cette barrière divise l'épithélium séminifère en deux espaces ou compartiments cellulaires : basal, audessous de la jonction occlusive continue, et adluminal, c'est-à-dire tourné vers la lumière du tube, au- dessus. Les cellules germinales les plus jeunes sont situées dans le compartiment basal, tandis que les cellules plus âgées, incluant les cellules méiotiques et postméiotiques telles les spermatides, occupent le compartiment adluminal. Seul le compartiment basal est perméable aux éléments sanguins. La raison d'être de cette barrière, son fonctionnement et son rôle dans l'achèvement de la spermatogenèse ont fait l'objet de multiples articles de synthèse très complets [5-7].

La question fondamentale que soulève la présence d'une barrière sélective aux éléments du sang dans l'épithélium séminifère est double. D'abord, comment les jonctions occlusives continues peuvent-elles interdire le passage de petites molécules provenant du sang tout en permettant celui de cellules germinales pourtant considérablement plus grandes ? Le déplacement des cellules germinales fait sans doute appel à une dynamique des jonctions cellulaires qu'il reste à explorer. Ensuite, pour que les changements de perméabilité jonctionnelle soient vraiment efficaces, ils doivent se produire à une période précise du développement des cellules germinales, ce qui implique l'existence d'un mécanisme de coordination entre les deux événements.

Rappelons-le, les complexes de jonctions réunissant les cellules de Sertoli sont constitués de jonctions occlusives, lacunaires et adhérentes. Chaque jonction est reliée à l'autre dans le temps et dans l'espace de sorte que, lorsqu'un type de jonction est affecté, les autres le seront très probablement. La figure $1 A$ représente de façon schématique la relation dans l'espace entre une cellule de Sertoli et différentes cellules germi- 
nales, ainsi que la distribution des différents types de jonctions intercellulaires depuis la base jusqu'à l'apex d'une cellule de Sertoli, observée sur coupe ultramince. La figure $1 B$, qui lui est adjacente, représente les spécialisations intramembranaires correspondant aux diverses jonctions observées avec la technique de cryodécapage qui consiste à fendre à froid la membrane cellulaire pour en révéler l'intérieur. Les jonctions occlusives sont représentées par des points de fusion entre les membranes plasmiques adjacentes (figure 1A) et par des cordons de particules intramembranaires ou cordons de fermeture représentés ici par des traits ou des lignes (figure 1B). Les jonctions occlusives sont distribuées le long de la membrane latérale et apicale de la cellule de Sertoli unissant des portions de cellules de Sertoli adjacentes ou des portions de cellules germinales à une cellule de Sertoli. Des études utilisant des traceurs de perméabilité visibles en cryodécapage ont démontré que la continuité du cordon de fermeture est ce qui confère l'imperméabilité à la jonction occlusive [8]. Dès lors, seule la jonction occlusive continue dont les cordons de fermeture ceinturent complètement la cellule de Sertoli constitue une barrière anatomique dans l'espace intercellulaire. Les jonctions occlusives discontinues ou ponctuelles, faites de cordons de fermeture interrompus ou sporadiques, représentent un obstacle local contournable et, de ce fait, ne forment pas une barrière efficace [8]. Ces jonctions occlusives discontinues correspondent à des périodes dans la vie des jonctions occlusives : elles se forment près de la base de la cellule de Sertoli, deviennent une barrière continue dans le tiers inférieur de l'épithélium et se démantèlent en fragments de jonctions occlusives vers le tiers supérieur de l'épithélium [8]. Cette modulation, c'est-à-dire la formation-déformation des jonctions occlusives, s'effectue dans la même direction que celle de la migration des cellules germinales qui passent du compartiment cellulaire basal au compartiment adluminal. Il reste à élucider le ou les facteur(s) responsable(s) de la formation et la déformation des jonctions occlusives

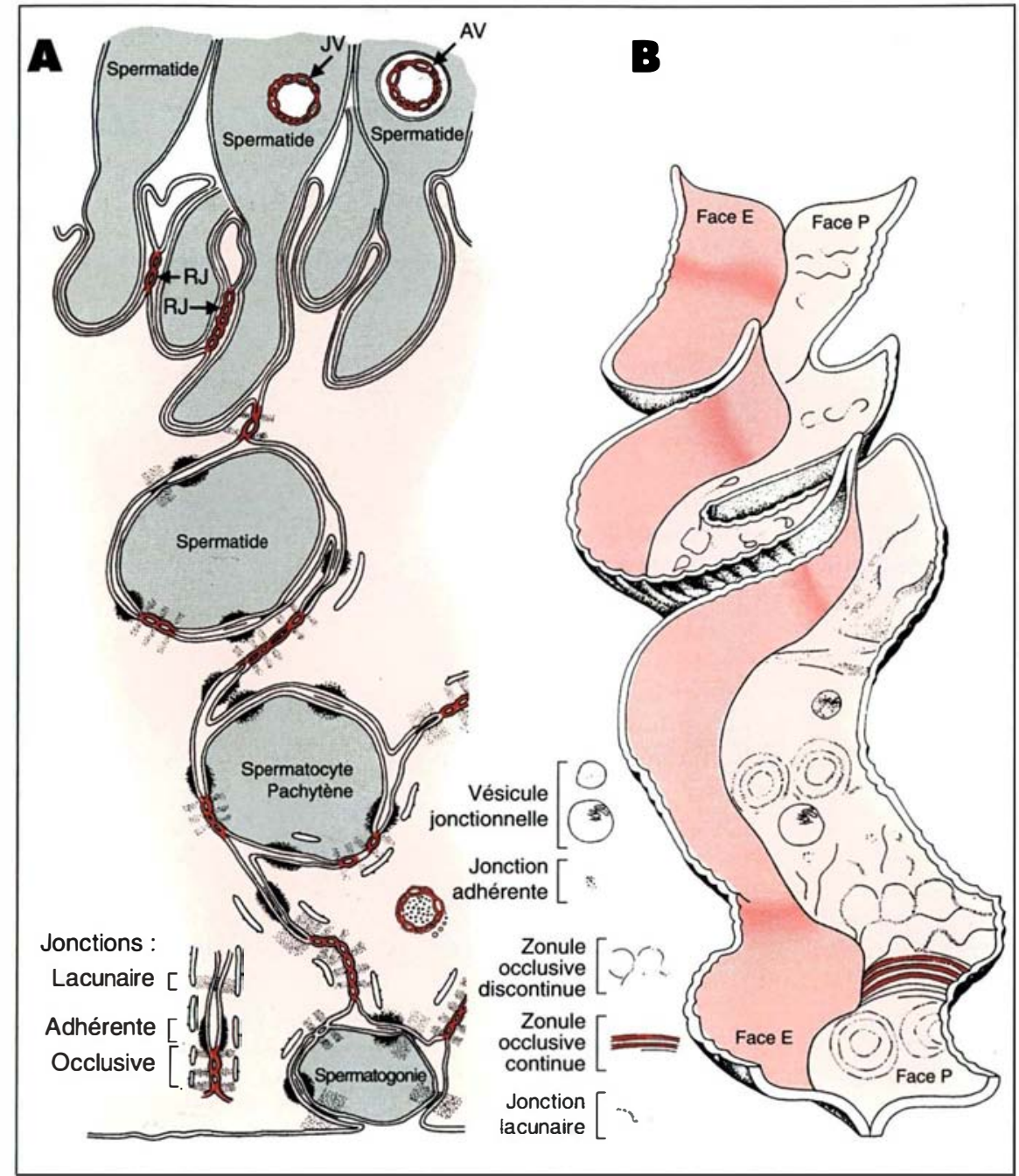

Figure 1. A. Vue schématique des jonctions intercellulaires établies le long de la membrane plasmique des cellules de Sertoli. Les jonctions lacunaires, adhérentes ou occlusives réunissent les membranes plasmiques, tantôt de cellules de Sertoli adjacentes, tantôt de cellules germinales (spermatogonies, spermatocytes, spermatides), avec celles de cellules de Sertoli. RJ : jonction occlusive réflexive. AV : vacuole autophagique dans lesquelles se trouvent des jonctions vétustes. JV : jonction occlusive annulaire vétuste. B. Membrane d'une cellule de Sertoli : vue d'une préparation de cryodécapage correspondant à la figure 1A. Seules les jonctions occlusives continues, appelées zonules pour indiquer le fait qu'elles entourent complètement la cellule de Sertoli, constituent la barrière hémato-testiculaire. Le démantèlement de ce type de zonules donne lieu aux zonules occlusives discontinues. Face $E$ : face externe, face $P$ : face plasmique. 
lules germinales. Il est étonnant de constater que la nature des particules intramembranaires qui constituent les cordons de fermeture des jonctions occlusives est encore peu connue. Les uns proposent qu'elle soit strictement protéique $[9,10]$, les autres lipoprotéique, à la fois protéique et lipidique $[11,12]$. Nous avons démontré par des techniques enzymatiques, que les membranes des complexes de jonction réunissant les cellules de Sertoli sont riches en cholestérol [13]. Récemment, une phosphoprotéine de surface de 225 kDa appelée ZO-1 (zonula occludens 1) a été identifiée aux sites des jonctions occlusives des cellules de Sertoli [14, 15]. Il semble que ce polypeptide, dont deux isoformes immunoréactives $\left(\mathrm{ZO}-1 \alpha^{+}\right.$et $\left.\mathrm{ZO}-\mathrm{l} \alpha\right)$ ont été obtenues par épissage alternatif d'ARN $[16,17]$, serve de lien transmembranaire entre les éléments de la jonction et la membrane plasmique. Par des techniques d'immunolocalisation, nous tentons de déterminer le rôle du polypeptide ZO-1 dans la distribution des particules intramembranaires des cordons de fermeture des jonctions occlusives. Une protéine de $160 \mathrm{kDa}$, la ZO-2, qui coprécipite avec la ZO-1, a été identifiée [18] et localisée [19] au site des jonctions occlusives avec la ZO-1. La cinguline, protéine de $140 \mathrm{kDa}$, a aussi été localisée par immunofluorescence au site des jonctions occlusives de plusieurs tissus, en particulier dans l'épididyme [20]. Dans l'épididyme et le canal déférent il existe une barrière hémato-tissulaire faite elle aussi de jonctions occlusives continues [21, 22]. Cependant, les barrières n'agissent pas d'une façon synchrone dans le testicule et dans ses canaux excréteurs [21, 22]. L'équipe de Tsukita a déterminé que la protéine ZO-1, généralement associée aux jonctions occlusives, se trouve aussi dans les jonctions adhérentes [23]. Cette même équipe a récemment purifié une protéine membranaire d'environ 65 kDa, l'occludine, qui est localisée exclusivement aux sites des jonctions des cellules épithéliales et endothéliales [24]. L'occludine et la ZO-2 sont deux protéines associées aux jonctions occlusives mais elles n'ont pas encore été localisées dans le testicule.

$\mathrm{m} / \mathrm{s} n^{\circ} 4$, vol. 11 , avril 95

\section{Les jonctions lacunaires}

Elles sont caractérisées par un espace de 2 à $4 \mathrm{~nm}$, entre deux membranes plasmiques adjacentes. En cryodécapage, les spécialisations intramembranaires correspondant à ce type de jonction sont de grosses particules disposées en rangées ou en plaques. L'analyse par diffraction de rayons $\mathrm{X}$ de la structure supramoléculaire de la jonction lacunaire révèle qu'elle est constituée d'un assemblage de canaux de communication, dont le faible diamètre limite l'accès des molécules [25]. Chaque canal est formé de six sous-unités protéiques appelées connexines [26]. L'injection d'anticorps anticonnexine 32 dans les larves de xénope empêche le transfert d'une cellule à l'autre de signaux qui règlent plusieurs étapes précises du développement [27]. Cela suggère que la jonction lacunaire jouerait un rôle dans la morphogenèse et la différenciation cellulaire. Enfin, une protéine de $16 \mathrm{kDa}$ décrite dans des préparations de jonctions lacunaires de plusieurs tissus appartient également, semble-t-il, à la $\mathrm{H}^{+}$- ATPase [28] .

En rapprochant les membranes plasmiques de cellules de Sertoli adjacentes, les jonctions occlusives ont certainement aussi un rôle de structuration de l'épithélium séminifère car elles attachent les cellules entre elles. La barrière peut être contournée par des transports actifs entre les cellules de Sertoli (endocytose, phagocytose ou autre). La fonction principale de la jonction occlusive est de fermer l'espace intercellulaire et, par conséquent, de l'exclure comme voie d'accès des molécules vers l'intérieur du tubule séminifère en contribuant à l'établissement de gradients dans l'épithélium. La jonction lacunaire n'est pas étanche [29] ; elle joue un rôle dans les échanges intercellulaires via des messages chimiques. Ces échanges de messages assurent la coordination des processus physiologiques entre les cellules qui se comportent comme si elles formaient un syncytium fonctionnel. La perméabilité des canaux de communication peut être adaptée aux exigences physiologiques rythmiques de la morphogenèse et de la différenciation cellulaire associées au cycle de l'épi- thélium séminifère en contrôlant le passage de molécules et d'ions d'une cellule à l'autre. Par exemple, l'élévation de la concentration de $\mathrm{Ca}^{2+}$ résultant d'une lésion cellulaire provoquerait la fermeture des canaux de communication [30, 31] et les changements de $\mathrm{pH}$ induiraient un découplage des cellules.

Outre son rôle dans les communications entre les cellules, la jonction lacunaire semble requise pour la formation et le démantèlement de la jonction occlusive et donc pour le fonctionnement de la barrière hémato-testiculaire [8, 32]. Chez l'adulte, la taille et le nombre des jonctions lacunaires sont inversement reliés à la longueur des cordons de particules qui forment les jonctions occlusives. On peut donc s'attendre à ce que le nombre et la fonction de la jonction lacunaire varient durant les périodes de renouvellement des jonctions occlusives de la cellule de Sertoli au cours du cycle de l'épithélium séminifère et aussi durant différentes phases d'activité spermatogénétique [32-34]. Quelques protéines de la famille de la connexine ont été localisées dans le testicule par immunofluorescence [35]. La technique d'immunoperoxydase a permis de montrer que la connexine 43 est présente entre les cellules de Leydig avant la naissance. Après la naissance, et simultanément avec le début de la spermatogenèse, cette protéine apparaît dans les tubules séminifères où sa distribution coïncide avec celle des jonctions lacunaires de la barrière hématologique du testicule [36]. L'état physiologique d'une jonction est généralement mesuré par sa compétence à établir des communications entre cellules. Pourtant, très peu de travaux ont étudié le couplage entre les cellules de Sertoli. Les travaux d'Eusebi [37] ont montré que ces cellules étaient électriquement couplées avant la puberté, mais pas après. Dans sa thèse, Spitzer [38] avait auparavant établi que les cellules de Sertoli étaient électriquement couplées entre elles et avec les cellules germinales et les cellules myoïdes. Ces deux études se sont déroulées avant l'avènement de la technique dite de "transillumination" [39] qui permet d'identifier le stage de développement des cellules ger- 
minales dans les tubes séminifères isolés, ce qui est nécessaire pour effectuer les études de couplage. Il est donc possible que les cellules de Sertoli ne soient électriquement couplées que pendant certaines phases du développement des cellules germinales.

On sait maintenant que les concentrations de testostérone $[40,41]$ et de FSH [42] à l'intérieur d'un segment de tubule séminifère varient en fonction du stade du cycle de l'épithélium séminifère auquel est ce segment. Les jonctions lacunaires étant particulièrement sensibles aux variations hormonales, la communication entre les cellules de Sertoli et leur couplage métabolique peut vraisemblablement varier d'un segment de tubule séminifère à un autre, selon les variations hormonales locales liées aux stades du cycle de l'épithélium séminifère. Les modifications des jonctions lacunaires sont donc elles-mêmes susceptibles d'influencer l'évolution des jonctions occlusives de la barrière hémato-testiculaire.

\section{Les jonctions adhérentes}

Troisième composante du complexe de jonctions de la cellule de Sertoli, elles sont présentes entre les cellules de Sertoli adjacentes, de même qu'entre les cellules de Sertoli et les cellules germinales (figure $1 \mathrm{~A}$ et $1 \mathrm{~B}$ ). Dans le testicule adulte, les jonctions intercellulaires apparaissent selon une séquence spatio-temporelle particulière [8] ; l'établissement d'une adhérence entre les cellules concernées est un préalable à la formation de jonctions occlusives [43, 44]. L'administration d'anticorps contre la cadhérine E, protéine de 120-124 $\mathrm{kDa}$ associée aux jonctions adhérentes, empêche la formation de nouvelles jonctions occlusives fonctionnelles et perturbe l'intégrité de celles déjà présentes [45]. La soustraction du $\mathrm{Ca}^{2+}$ du milieu extracellulaire modifie les propriétés adhésives des cadhérines, entraînant à la fois l'ouverture des jonctions occlusives et le détachement de la ZO-1 et de la cinguline des sites de jonctions [46]. Tsukita et son équipe ont découvert que la ZO-1 s'associe spécifiquement aux jonctions occlusives et aux jonc- présence de cadhérines [47]. De Philip et son groupe [48] ont montré que la période d'expression par les cellules de Sertoli des trois molécules d'adhérence, cadhérines épithéliale (E) (120-124 kDa), neuronale (N) $(127 \mathrm{kDa})$ et placentaire (P) (118 $\mathrm{kDa})$, est maximale deux semaines environ avant la mise en place des jonctions occlusives continues. Il reste à savoir si les cellules germinales expriment les mêmes molécules d'adhérence et si elles le font en même temps que les cellules de Sertoli.

L'expression de chaque cadhérine est réglée dans l'espace et dans le temps au cours du développement cellulaire. Ainsi, la molécule d'adhérence de la famille des cadhérines ex primée au début du développement des cellules germinales n'est pas nécessairement la même que celle qui est exprimée au milieu ou à la fin. Sur la même cellule de Sertoli, les molécules d'adhérence exprimées près de la base, face aux spermatogonies ou aux jeunes spermatocytes I, pourraient promouvoir la formation des jonctions occlusives, tandis que celles exprimées au niveau des spermatocytes du stade zygotène, situés un peu plus apicalement le long de la cellule de Sertoli, pourraient en favoriser le démantèlement. Comme les cellules de Sertoli font face en même temps à d'autres cellules de Sertoli et à des cellules germinales, des molécules d'adhérence homotypiques et hétérotypiques doivent co-exister dans l'épithélium séminifère. L'alternance de l'expression de l'un ou de l'autre type de molécules d'adhérence par les cellules de Sertoli et par les cellules germinales pourrait être à l'origine de changements morphologiques et fonctionnels des jonctions adhérentes d'abord, puis des jonction occlusives continues. Ce mécanisme pourrait assurer que les changements fonctionnels de la barrière hémato-testiculaire s'effectuent en coordination avec le développement des cellules germinales.

\section{Conclusion}

Dans le testicule, il existe une barrière hémato-testiculaire à l'origine de l'établissement de gradients et de la compartimentation de l'épithélium.
Pour comprendre le fonctionnement de cette barrière, nous avons analysé chacun des trois types de jonctions qui y sont présentes : occlusive, lacunaire et adhérente. Sur la membrane plasmique des cellules de Sertoli, les jonctions apparaissent selon une séquence spatio-temporelle définie. De sorte que le type de jonction formé influence la formation du type de jonction qui suit. La formation et la déformation de ces jonctions occlusives doivent s'effectuer en coordination avec le développement des cellules germinales en migration vers l'apex de l'épithélium. Il est possible que l'expression de molécules de connexine et d'adhérence, qui sont à l'origine de la formation des jonctions occlusives, assure cette coordination

Remerciements

Ce travail a été rendu possible grâce à l'octroi MT-1161 du Conseil canadien de la recherche médicale. I.'auteur exprime ici sa gratitude aı Dr María L.eiza Vitale pour ses suggestions au cours de la rédaction de cet article.

\section{R.-Marc Pelletier}

Département d'anatomie et de neurobiolo gie, faculté de médecine, université d'Ottawa, 451 Smyth Road, Ottawa, Ontario K1H 8M5, Canada.

\section{RÉFÉRENCES}

1. Elftman H. Sertoli cells and testis structure. Am J Anat 1963 ; 113 : 25-32.

2. Setchell BP, Volglmayr JK, Waites GMH. A blood-testis barrier restricting passage from blood into rete testis fluid but not into lymph. J Physiol 1969 ; 200 : 73-85.

3. Kormano M. Dye permeability and alkaline phosphatase activity of testicular capillaries in the postnatal. Histochemie 1967 ; 9 . 327-38.

4. Dym M, Fawcett DW. The blood-testis barrier in the rat and the physiological compartmentation of the seminif erous epithepartmentation of the seminif ero

5. Abraham M. The male germ cell protective barrier along phylogenesis. Int Rev (jytol 1991 ; 130 : 1119-90. 
6. Pelletier RM, Byers SW. The blood-testis barrier and Sertoli cell junctions : structural considerations. Microsc Res Tech $1992 ; 20$ : 3 33.

7. Jégou B. The Sertoli-germ cell communication network in mammals. Int Rev Cytol $1993 ; 147: 25-96$

8. Pelletier RM, Friend DS. The Sertoli cell junctional complex : structure and permeability to filipin in the neonatal and adul guinea pig. AmJ Anat 1983 ; 168 : 213-28.

9. Cereijido M, Robbins ES, Dolan WJ, Rotunno CA, Sabatini DD. Polarized monolayers formed by epithelial cells on a permeable and transluscent support. J Cell Biol $1978 ; 77: 853-80$.

10. Meza I, Sabenero M, Stefoni E, Cereijido $M$. Occluding junctions and cytoskeletal components in a cultured transporting epithelium. J Cell Biol $1982 ; 87$ : 746-54.

11. Pinto da Silva $P$, Kachar B. On tight junction structure. Cell $1982 ; 28: 441-50$.

12. Kan FWK. Cytochemical evidence for the presence of phospholipids in epithelial tight junction strands. J Histochem Cytochem $1993 ; 41: 649-56$.

13. Pelletier RM, Vital ML. Filipin $v s$ enzy matic localization of cholesterol in Guine pig, mink and mallard duck testicular cells J Histochem Cytochem 1994; 42: 1539-54.

14. Stevenson BR, Siliciano JD, Mooseker MS, Goodenough DA. Identification of ZO$1:$ a high molecular weight polypeptide associated with the tight junction (zonula occludens) in a variety of epithelia. I Cell Biol $1986 ; 103$ : 755-66.

15. Anderson JM, Stevenson BR, Jesaitis LA Goodenough DA, Mooseker MS. Characterization of ZO-1, a protein component of tight junction from mouse liver and MadinDarbin canine kidney cells. J Cell Biol 1988 ; $106: 1141-9$.

16. Willott E, Balda MS, Heintzelman M, Jameson B, Anderson JM. Localization and differential expression of two isoforms of the tight junction protein ZO-1. Am J Physiol 1992 ; 262 (Cell Physiol , 31) : C1119-24.

17. Balda MS, Anderson JM. Two classes of tight junctions are revealed by $\mathrm{ZO}-1$ isoforms. Am J Physiol 1993 ; 264 (Cell Physiol, 33) : C918-24.

18. Gumbiner B, Lowenkopf T, Apatira D. Identification of a $160 \mathrm{kDa}$ polypeptide that binds to the tight junction protein $\mathrm{ZO}-1$. Proc Natl Acad Sci USA 1991 ; 88 : 3460-4.

19. Jesaitis LA, Goodenough DA. Molecular characterization and tissue distribution of $\mathrm{ZO}-2$ a tight junction protein homologous to ZO-1 and the drosophila discs-large tumor suppressor protein. J Cell Biol 1994 ; $124: 949-61$

20. Byers SW, Citi S, Anderson JM, Hoxter B. Polarized functions and permeability properties of rat epididymal epithelial cells in vitro. J Reprod Fert $1992 ; 95: 385-96$
21. Pelletier RM. Blood barriers of the epididymis and vas deferens act asynchronously with the blood barier of the testis in the mink (Mustela vison). Microsc Res Tech 1994 $27: 333-49$.

22. Pelletier RM. A freeze-fracture study of cell junctions in the epididymis and vas deferens of a seasonal breeder : the mink (mustela vison). Microsc Res Tech 1995; $30: 37$. 53.

23. Itoh $M$, Nagafuchi A, Yonemura S, Kitani-Ysuda T, Tsukita Sa, Tsukita Sh. The 220 $\mathrm{KD}$ protein colocalizing with cadherins in non-epithelial cell is identical to $\mathrm{ZO}-1$, a tight junction-associated protein in epithelial cells : cDNA cloning and immunoelectron microscopy. J Cell Biol 1993 ; 121 : 491502

24. Furuse MS, Hirase T, Itoh M, Nagafuch A, Yonemura S, Tsukita S, Tsukita Sh. Occludin : a novel integral membrane protein localizing at tight junctions. J Cell Biol 1993 . $123: 1777-88$.

25. Makowsky L, Caspar DL, Philips WC Goodenough DA. Gap junction structures. 2. Analysis of the X-ray diffraction data. $J$ Cell Biol 1977 ; 74 : 629-45.

26. Haefliger JA, Bruzzone R, Jenkins NA, Gilbert DJ, Copeland NG, Paul DL. Four novel members of the connexin family of gap junction protein : molecular cloning expression and chromosome mapping. Biol Chem 1992 ; 267 : 2057-64.

27. Warner AE, Guthrie SC, Gibula NB. Antibodies to gap junction protein selectively disrupt junctional communication in early amphibian embryo. Nature $1984 ; 311$ : 127 31.

28. Dermietzel R, Volker M, Hwang TK Berzborn RJ, Meyer HE. A $16 \mathrm{kDa}$ protein co-isolating with gap junctions from brain tissue belonging to the class of proteolipids of the vacuolar $\mathrm{H}^{+}$-ATPase. FEBS Lett 1989 $253: 1-5$

29. Revel JP, Karnovsky MJ. Hexagonal array of subunits in intercellular junctions of the mouse heart and liver. J Cell Biol 1967; 33 C7-12.

30. Oliviera-Castro GM, Loewenstein WR. Junctional membrane permeability : effect of divalents cations. J Membr Biol 1971 ; 73 . 69-89.

31. Spray DC, White RL, Campis de Cavalho AC, Harris AL, Bennett MVL. Gating of gap junction channels. Biophys J $1984 ; 45$ : 219 jun.

32. Pelletier RM. Cyclic modulation of Sertoli cell junctional complexes in a seasonal breeder : the mink (Mustela vison). Am J Anat 1988 ; $183: 68-102$.

33. Pelletier RM. A novel perspective : the occluding zonule encircles the apex of the Sertoli cell as observed in birds. Am J Anat $1990 ; 188: 87-108$.

34. Pelletier RM. The effects of long-term experimental cryptorchidism on the bloodtestis barrier in adult guinea pigs. $J$ Androl $1993 ; 14$ (suppl) : 56 .
35. Risley MS. Cell age and stage dependent distribution of connexin 43 gap junction in testis. J Cell Sci $1992 ; 103: 81-96$.

36. Pelletier RM. The temporal changes in the distribution of connexin 43 in continual (Guinea pig) and seasonal breeders (mink) testes are associated with the germ cell differentiation and the modulation of the Sertoli cell junctional barrier. J Androl 1995 (sous presse)

37. Eusebi F, Ziparo E, Fratamico G, Russo $M$ Stefanini $M$ Intercellular communication in the rat seminiferous tubules. Dev Biol 1983 ; 100 : 249-55.

38. Spitzer NC. Low resistance junctions in male germinal tissues of lily and the rat. $\mathrm{Ph}$ D. Dissertation, Harvard University, 1969.

39. Paroinen M, Ruokonen A. Endogenous steroids in the rat seminiferous tubules Comparison of the state of the epithelia cycle isolated by transillumination-assisted micro dissection. J Androl 1982 ; 3 : 211-20.

40. Parvinen M, Nikula $H$, Huhtaniemi I. Cyclic regulation of rat Leydig cell testosterone production by seminiferous tubules. Ann NY Acad Sci 1984 ; 438 : 681-3.

41. Parvinen M, Huhtaniemi I. Testosterone micro milieu in staged rat seminiferous tubules. J Steroid Biochem $1990 ; 36$ : 377-81

42. Heckert L, Griswald D. Expression of FSH receptors in RNA in rat testis and Sertoli cells. Mol Endocrinol $1991 ; 5: 670-7$.

43. Takeichi M. Cadherins, a molecular family essential for a selective cell-cell adhesion and animal morphogenesis. Trends Genet $1987 ; 3: 213-7$

44. Edelman GM. Modulation of cell adhesion during induction, histogenesis, and perinatal development of the nervous system. Annu Rev Neurosci $1984 ; 7: 339-77$

45. Gumbiner B, Stevenson B, Grimaldi A The role of the cell adhesion molecule Uvomorulin in the formation and maintenance of the epithelial junctional complex. J Cell Biol 1988 ; 107 : 1575-87.

46. Siliciano JD, Goodenough DA. Localization of the tight junction protein, $\mathrm{ZO}-1$, is modulated by extra cellular calcium and cell-cell contact in Madin-Darbin canine kidney epithelial cells. J Cell Biol 1988 ; 107 : $2389-99$.

47. Itoh M, Yonemura S, Nagafuchi A, Tsukita S, Tsukita Sh. A protein : its specific localization at cadherin-based cell-cell adhesion sites. J Cell Biol 1991 ; 115 : 1449-62.

48. Wu JC, Gregory CW, DePhilip RM. Expression of E-Cadherin in immature rat and mouse testis and in rat Sertoli cell cultures. Biol Reprod 1993 ; 49 : 1353-61.

\section{TIRÉS À PART}

R.M. Pelletier. 